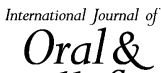 Maxillofacial Surgery \\ Clinical Paper \\ Orthognathic Surgery}

\section{Three-dimensional printed final occlusal splint for orthognathic surgery: design and validation}

\begin{abstract}
E. Shaheen, Y. Sun, R. Jacobs, C. Politis: Three-dimensional printed final occlusal splint for orthognathic surgery: design and validation. Int. J. Oral Maxillofac. Surg. 2016; $x x x: x x x-x x x$. (C) 2016 International Association of Oral and Maxillofacial

Surgeons. Published by Elsevier Ltd. All rights reserved.
\end{abstract}

\begin{abstract}
Orthognathic surgery is indicated for the treatment of significant skeletal malocclusion and is normally prepared using conventional face bows combined with two-dimensional cephalometric analysis and manually made splints. With recent developments in oral imaging, more orthognathic surgeries are being planned using three-dimensional computer-aided design and manufacturing (3D CAD/ CAM) software. The purpose of this study was to present a protocol for the design and 3D printing of final digital occlusal splints based on 3D planning of orthognathic surgery and to validate the accuracy of these splints. The $3 \mathrm{D}$ virtual planning was performed in PROPLAN software (Materialise). The required data were then exported into 3-matic software (Materialise) to design the splints, which were 3D printed in biocompatible material using an Objet Connex 350 printer (Stratasys). To validate the accuracy of the splints, the cases of 20 patients undergoing orthognathic surgery were analysed. The splints were assessed clinically and quantitatively by comparing the printed splints to the conventional analogue set-up (clinical standard) and recording the absolute distance errors of three landmarks. The mean absolute distance error was $0.4 \mathrm{~mm}$ (standard deviation $0.17 \mathrm{~mm}$ ), which falls within clinically accepted error margins. The absolute distance error ranged from 0.12 to $0.88 \mathrm{~mm}$.
\end{abstract}

\author{
E. Shaheen ${ }^{1,2}$, Y. Sun ${ }^{1,2}$, \\ ${ }^{1}$ OMFS IMPATH Research Group, \\ Department of Imaging and Pathology, \\ Faculty of Medicine, KU Leuven, Leuven, \\ Belgium; ${ }^{2}$ Department of Oral and \\ Maxillofacial Surgery, University Hospitals \\ Leuven, Leuven, Belgium
}

Key words: virtual surgical planning; orthognathic surgery; wafer; splint; 3D printing; CAD/ CAM.

Accepted for publication 7 October 2016
Successful orthognathic surgery implies the achievement of the correct occlusal relationship, as well as facial balance and harmonization. Splints are used in orthognathic surgery to transfer the preoperative surgical plan and to reposition the jaws into the required optimised occlusion. ${ }^{1}$

Recent developments in dentomaxillofacial imaging using computed tomography (CT) and cone beam CT (CBCT) have allowed further developments in computer-aided design and manufacturing (CAD/CAM) software, leading to a variety of algorithms and software for threedimensional (3D) virtual planning of orthognathic surgery. ${ }^{2-5}$ The main advantages of $3 \mathrm{D}$ planning include the production of a virtual skull and dentition and the direct production of digital and subsequent physical splints, ${ }^{6}$ leading to more accurate planning, time savings, and better results.

Previous research has investigated the accuracy of final digital splints in smallscale validation studies with a maximum of 10 cases, ${ }^{3,6,7}$ or simply through proof of concept. ${ }^{8}$ Comparisons were made to conventional manual splints and considered as the ground truth, which is not entirely correct. Some comparison studies have 
reported mean values, ${ }^{9}$ which may underestimate the magnitude of the error, and others have reported the absolute errors. ${ }^{8}$

The aims of this study were to present the steps leading to the design and printing of final digital occlusal splints based on 3D planning of orthognathic surgery and to validate the accuracy of these final digital splints in a larger scale study by comparing the printed splints to the conventional analogue set-up (clinical standard) in terms of absolute errors.

\section{Materials and methods}

\section{Virtual 3D planning protocol}

The virtual planning protocol for orthognathic surgery was implemented in PROPLAN software version 2.1 (Materialise, Leuven, Belgium). Preoperative imaging was performed with a 128 -slice multi-slice spiral CT scanner (Siemens Somatom Definition Flash; Siemens AG, Erlangen, Germany) with settings of $120 \mathrm{kV}$, $161 \mathrm{mAS}$, and slice thickness $0.75 \mathrm{~mm}$. DICOM (Digital Imaging and Communications in Medicine) images of the patient were imported into the project and the different components segmented (mandible, maxilla, skull) using a thresholding method. The plaster casts of the upper and lower dentitions were scanned with a highresolution optical scanner (Activity 885; SmartOptics, Bochum, Germany) and the images saved as stereolithography (STL) files. These casts were registered to the corresponding upper and lower jaws via point-based registration to form composite models of the maxilla and the mandible. ${ }^{10}$ The plaster casts were put into the final required position by the surgeon (occlusion cast) and scanned by CBCT. The DICOM images of the occlusion cast were imported into a new PROPLAN project, where a $3 \mathrm{D}$ object was made. The virtual osteotomies were then performed and the $3 \mathrm{D}$ object of the occlusion cast was registered to the upper jaw and the cut lower jaw was registered to the registered occlusion cast via point-based registration. The registered casts were then exported from the PROPLAN project and imported into 3-matic software (version Medical 10; Materialise) to design the final splint. This was then $3 \mathrm{D}$ printed in biocompatible material using an Objet Connex 350 printer (Stratasys, Eden Prairie, MN, USA) with a slice thickness of $0.03 \mathrm{~mm}$.

When this protocol was first implemented, inaccuracies were encountered during the production of the final splints, leading to an inaccurate final occlusion when compared to the final splints made manually
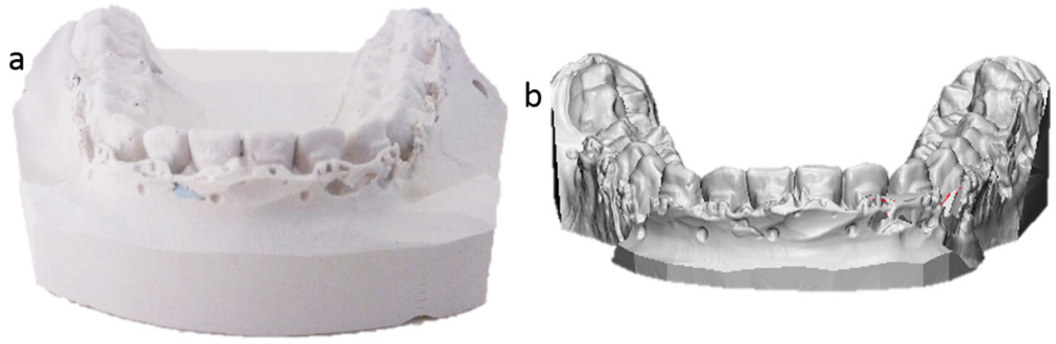

Fig. 1. (a) Plaster cast with drilled 1-mm holes. (b) The same cast scanned with a highresolution optical scanner. and produced in the dental laboratory. Further investigations revealed that the errors in the final splints resulted from a lack of landmarks for the point-based registration and differences in resolution between the occlusion cast scanned by CBCT and the high resolution of the optical scanner $(6 \mu \mathrm{m})$.

\section{Improved protocol}

In order to overcome these issues, the following alterations were made: (1) holes of $1 \mathrm{~mm}$ in diameter were drilled into the casts away from the dentition, covering the full arch, prior to the scans. These served as landmarks (Fig. 1). (2) A special acquisition protocol for scanning the casts by CBCT was used. When different protocols for scanning the occlusion casts were investigated, it was found that only this protocol provided fairly visible visualisation of the landmarks. Table 1 and Fig. 2 provide the acquisition settings for the protocol used (special cast protocol) and another high-dose protocol developed for patients (high-dose patient protocol). (3) The registration step was then modified to the use of landmarks distributed across the full arch (minimum of four landmarks: one posterior left, one posterior right, and two in the anterior canine-to-canine region).

\section{Validation study}

A validation study was conducted to evaluate the accuracy of the final splints produced with the newly proposed protocol. Twenty patient cases were included in the study, with 14 prepared for bilateral sagittal split osteotomy (BSSO) surgery and the other six planned for bimaxillary surgery (BSSO and Le Fort I).

Ethical approval was obtained from the necessary ethics review board. No informed consent was required, as no patient identifiable data were disclosed. All testing and examinations were done on plaster casts.

Every case was assessed clinically by the surgeon performing the operations by checking the relative positions of the midlines of the upper and lower jaws and the contact points, as in the original occlusion cast. Furthermore, a quantitative evaluation based on the analysis of distance errors between landmarks was performed.

For each case, the casts used for splint production were mounted on the printed digital splint and scanned with the special cast CBCT scan protocol (referred to as scan 2), which was identical to the scan of the original occlusion cast (referred to as scan 1). The DICOM images of both scans were then imported into Amira software (FEI, Hillsboro, OR, USA). Scan 2 data were registered onto the lower jaw of scan 1 via voxel-based registration with mutual information. $^{11-13}$ The 3D object of the registered cast was then exported as an STL file and imported into the PROPLAN project of the occlusion cast. Three landmarks were identified on each upper cast
Table 1. Acquisition settings for the CBCT protocols used.

\begin{tabular}{lll}
\hline & \multicolumn{1}{c}{ Special cast protocol } & High-dose patient protocol \\
\hline CBCT system & Planmeca Promax 3D Max & Planmeca Promax 3D Max \\
Tube current (mA) & 12.5 & 7.1 \\
Gray scale (bit) & 12 & 12 \\
Potential (kV) & 80 & 96 \\
Exposure type & Pulsed & Pulsed \\
Scan time (s) & 22.5 & 15 \\
Voxel size (mm) & 0.10 & 0.15 \\
FOV (mm) & $100 \times 93$ & $80 \times 50$ \\
Detector type & Flat panel & Flat panel \\
\hline
\end{tabular}

CBCT, cone beam computed tomography; FOV, field of view. 

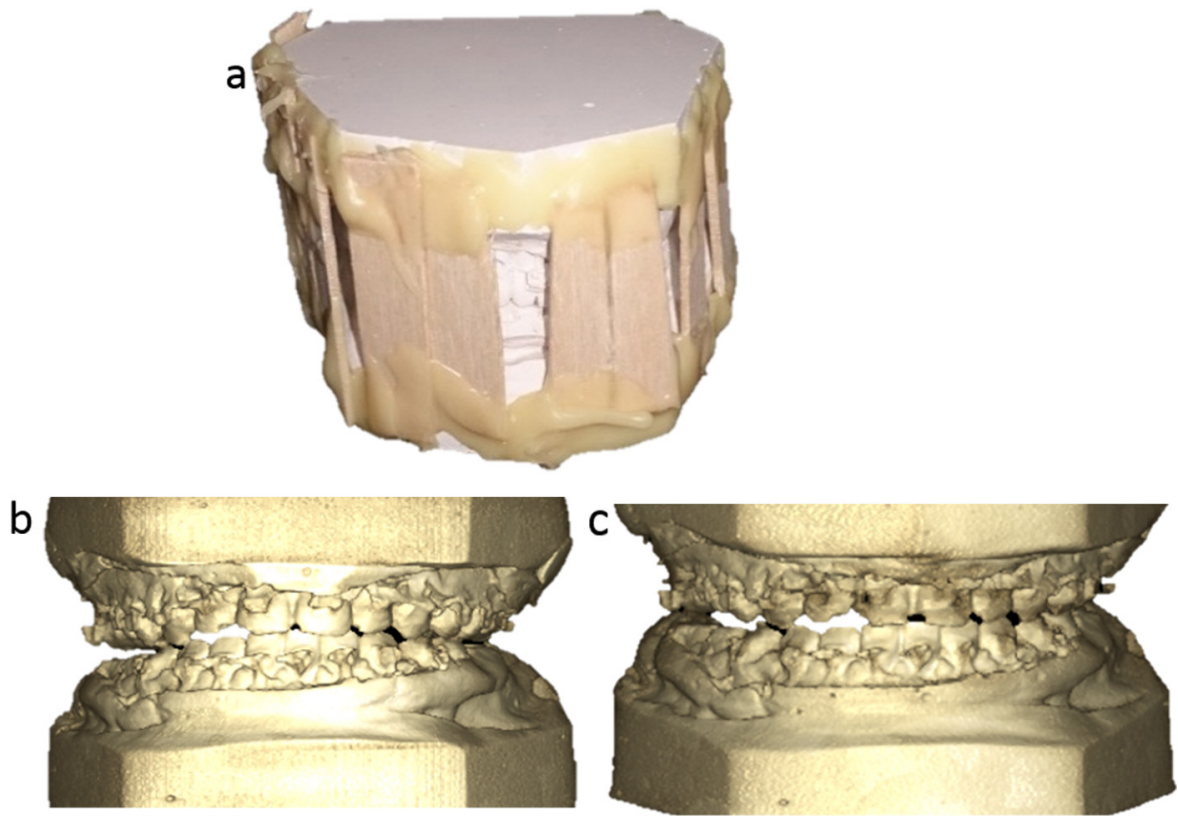

Fig. 2. (a) Occlusion cast as prepared by the surgeon. (b) Occlusion cast scanned with the special cast CBCT protocol. (c) The same occlusion cast scanned with the high-dose patient CBCT protocol, as described in Table 1.

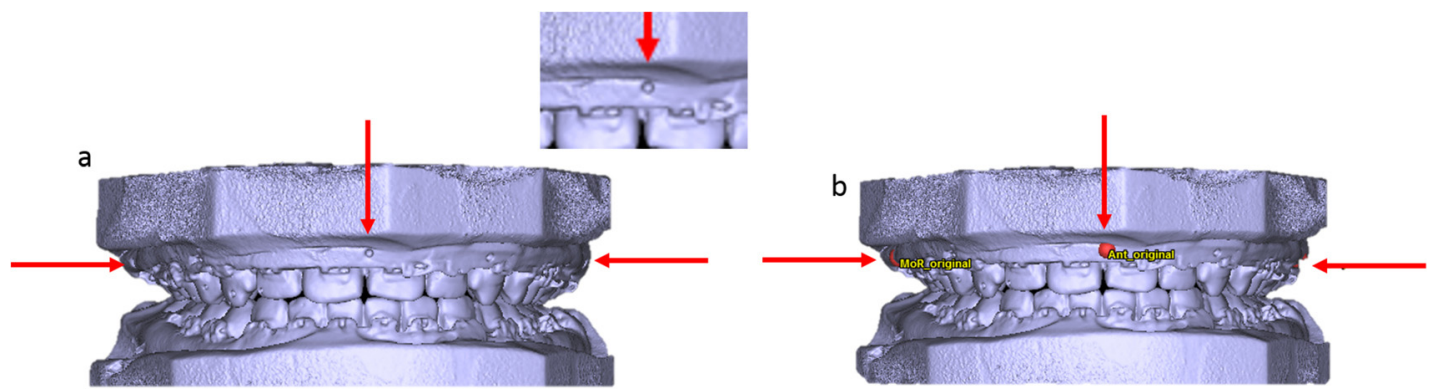

Fig. 3. (a) Three landmarks on the upper cast from the drilled holes: anterior region, posterior left, and posterior right (indicated with red arrows). (b) The same landmarks identified by red spheres for measurements (indicated with red arrows) (For interpretation of the references to colour in this figure legend, the reader is referred to the web version of this article.).

from the drilled holes: anterior region, posterior left, and posterior right, as shown in Fig. 3. The distances between each landmark on the upper cast of the occlusion cast and the corresponding landmark on the digital splint cast were calculated (Fig. 4), resulting in a total of 60 distance errors.

\section{Results}

\section{Clinical evaluation results}

All 20 cases were evaluated by the same surgeon performing the operations, and the accuracy of the final splints was considered clinically acceptable .

\section{Quantitative evaluation results}

Table 2 shows the absolute differences between the landmarks (distance errors) on the upper occlusion cast and the corresponding landmarks on the upper cast with the digital splint after superimposition of the lower jaw casts, as described in the validation study.

The mean absolute distance error was $0.4 \mathrm{~mm}$ with a standard deviation of $0.17 \mathrm{~mm}$. The absolute distance error ranged from 0.12 to $0.88 \mathrm{~mm}$.

\section{Discussion}

The aim of this study was to present a protocol to accurately produce $3 \mathrm{D}$ printed final splints for use in the positioning of the bone segments during orthognathic surgery. Shqaidef et al. recently presented a validation study in which rapid prototyped final orthognathic surgical splints were compared to conventional wafers. ${ }^{8}$ They reported absolute mean distances ranging from $0.04 \mathrm{~mm}$ to $1.73 \mathrm{~mm}$, with a mean value of $0.94 \mathrm{~mm}$, which was considered to be clinically acceptable. In the present study, the upper limit of the absolute distance error was $0.88 \mathrm{~mm}$, which falls within the clinically acceptable range. Moreover, the mean absolute distance error was $0.4 \mathrm{~mm}$, which is considered an improvement.

The validation procedure relied on voxel-based registration rather than point-based registration, which overcomes the human errors that may possibly be introduced during point-based registration, thereby leading to more accurate comparison results. Furthermore, the occlusion cast was used to test the accuracy of the final splint as the gold standard reference instead of the conventional splint, as reported in previous studies. 3

The routinely applied clinical protocol was used as standard. This consisted of fixing the occlusion cast through the 


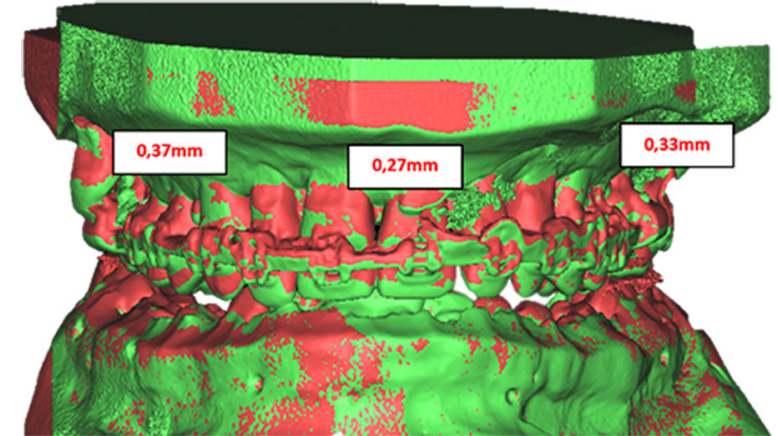

Fig. 4. Occlusion cast (green) and cast with the digital splint (red) superimposed based on the lower jaw; the distances between landmarks were calculated. In this example, distance errors for the anterior, posterior left, and posterior right were $0.27 \mathrm{~mm}, 0.33 \mathrm{~mm}$, and $0.37 \mathrm{~mm}$, respectively (For interpretation of the references to colour in this figure legend, the reader is referred to the web version of this article.).

application of wax and wooden sticks (Fig. 2). Therefore, CBCT scans were needed to obtain digital models of the casts. As a result, and due to the limited resolution of the CBCT scan (to $0.1 \mathrm{~mm}$ ), as expected, the minimum distance error reported was $0.12 \mathrm{~mm}$. An alternative to this fixation would be the use of glue or wax, but this was not tested in the present study.

The currently used preoperative protocol relies on preoperative CT. The mean effective dose for this head CT protocol is reported to be $1.8 \mathrm{mSv}$ based on internal medical physics reports. A number of CBCT scanners that have the ability to scan the full skull have recently been introduced to the market. Two such CBCT

systems have been tested in the study hospital; these have mean effective doses of $0.3 \mathrm{mSv}$ and $1 \mathrm{mSv}$, respectively, for the full skull scan protocol. With this obvious benefit to the patient of a lower radiation dose, the use of CBCT instead of CT for preoperative imaging is currently being validated; however, this was not included in the present study.

The use of point-based registration combined with landmarks scanned at high resolution was the main feature of the protocol used to produce the final surgical occlusal splints. This technique produced errors of up to $0.88 \mathrm{~mm}$. Thus, the use of an accurate intraoral scanner to directly scan the teeth and the use of 3D printed models to directly form the occlusion cast

Table 2. Absolute differences in distances (distance errors) for the three landmarks (anterior, posterior left, and posterior right) between the upper cast of the occlusion cast and the corresponding cast with the final digital splint for 20 cases. The mean and standard deviation (SD) values are also reported for each landmark.

\begin{tabular}{llcc}
\hline Case number & Anterior $(\mathrm{mm})$ & Posterior left $(\mathrm{mm})$ & Posterior right $(\mathrm{mm})$ \\
\hline 1 & 0.34 & 0.36 & 0.29 \\
2 & 0.27 & 0.33 & 0.37 \\
3 & 0.58 & 0.35 & 0.49 \\
4 & 0.25 & 0.6 & 0.63 \\
5 & 0.21 & 0.3 & 0.57 \\
6 & 0.16 & 0.19 & 0.13 \\
7 & 0.18 & 0.38 & 0.26 \\
8 & 0.33 & 0.18 & 0.12 \\
9 & 0.33 & 0.37 & 0.52 \\
10 & 0.28 & 0.58 & 0.27 \\
11 & 0.35 & 0.57 & 0.24 \\
12 & 0.69 & 0.33 & 0.14 \\
13 & 0.31 & 0.58 & 0.39 \\
14 & 0.55 & 0.59 & 0.55 \\
15 & 0.52 & 0.28 & 0.36 \\
16 & 0.49 & 0.33 & 0.25 \\
17 & 0.76 & 0.88 & 0.63 \\
18 & 0.18 & 0.73 & 0.41 \\
19 & 0.43 & 0.68 & 0.48 \\
20 & 0.32 & 0.42 & 0.38 \\
Mean (SD) & $0.38(0.17)$ & $0.45(0.18)$ & $0.37(0.16)$ \\
\hline
\end{tabular}

would be beneficial, and the error would decrease to the resolution of the scanner.

The Objet 3D printer with biocompatible material (MED610) is medically approved for the temporary intraoral application of surgical guides and devices for up to $24 \mathrm{~h}$. This was the only $3 \mathrm{D}$ printer used in this validation study. Previous research has already validated the use of stereolithography (SLA-250/50 machine, 3D-Systems, USA) with layers $0.1 \mathrm{~mm}$ thick as an acceptable method for rapid prototyping of the final splint. ${ }^{8}$

A protocol for virtual 3D planning of orthognathic surgery with a focus on final occlusal splints is presented herein. The validation study proved the high accuracy of these 3D printed splints for use during orthognathic surgery: the maximum error was $0.88 \mathrm{~mm}$ and the mean error was $0.4 \mathrm{~mm}$, which is an improvement on the results of other studies and is lower than the clinically relevant error margin of $0.5 \mathrm{~mm}$. This protocol can be used to produce accurate 3D final occlusal splints with clinically acceptable results.

\section{Funding}

None.

\section{Competing interests}

Nothing to declare.

\section{Ethical approval}

Ethical approval was obtained from the Ethics Review Board of the University Hospitals Leuven (S57587).

\section{Patient consent}

Informed consent was not required, as no patient identifiable data are disclosed. All testing and examinations were done on plaster casts.

\section{References}

1. Metzger MC, Hohlweg-Majert B, Schwarz U, Teschner M, Hammer B, Schmelzeisen R. Manufacturing splints for orthognathic surgery using a three-dimensional printer. Oral Surg Oral Med Oral Pathol Oral Radiol Endod 2008;105:e1-e7.

2. Okumura H, Chen LH, Yokoe Y, Tsutsumi S, Oka M. CAD/CAM fabrication of occlusal splints for orthognathic surgery. J Clin Orthod 1999;33:231-5.

3. Gateno J, Xia J, Teichgraeber JF, Rosen A, Hultgren B, Vadnais T. The precision of computer-generated surgical splints. J Oral Maxillofac Surg 2003;61:814-7. 
4. Swennen GR, Mollemans W, De Clercq C, Abeloos J, Lamoral P, Lippens F, et al. A cone-beam computed tomography triple scan procedure to obtain a three-dimensional augmented virtual skull model appropriate for orthognathic surgery planning. I Craniofac Surg 2009;20:297-307.

5. Xia JJ, Gateno J, Teichgraeber JF. A new clinical protocol to evaluate cranio-maxillofacial deformity and to plan surgical correction. J Oral Maxillofac Surg 2009;67: 2093-106.

6. Zinser MJ, Mischkowski RA, Sailer HF, Zöller JE. Computer-assisted orthognathic surgery: feasibility study using multiple CAD/CAM surgical splints. Oral Surg Oral Med Oral Pathol Oral Radiol 2012;113: 673-87.

7. Adolphs N, Liu W, Keeve E, Hoffmeister B. RapidSplint: virtual splint generation for orthognathic surgery - results of a pilot series. Comput Aided Surg 2014;19:20-8.
8. Shqaidef A, Ayoub AF, Khambay BS. How accurate are rapid prototyped (RP) final orthognathic surgical wafers? A pilot study. Br J Oral Maxillofac Surg 2014;52:609-14.

9. Kim BC, Lee CE, Park W, Kim MK, Zhengguo P, Yu HS, et al. Clinical experiences of digital model surgery and the rapid-prototyped wafer for maxillary orthognathic surgery. Oral Surg Oral Med Oral Pathol Oral Radiol Endod 2011;111:278-85.

10. Lin X, Chen T, Liu J, Jiang T, Yu D, Shen SG. Point-based superimposition of a digital dental model on to a three-dimensional computed tomographic skull: an accuracy study in vitro. $\mathrm{Br} J$ Oral Maxillofac Surg 2015;53:28-33.

[11] Viola P, Chao A. Multiple sensor image alignment by maximization of mutual information. Massachusetts Institute of Technology and ALPHATECH, Inc.; 1995

12. Collignon A, Maes F, Delaere D, Vandermeulen D, Suetens P, Marchal G. Automated multi-modality image registration based on information theory. Inf Process Med Imaging 1995;3:263-74.

13. Pluim JP, Maintz JA, Viergever MA. Mutualinformation-based registration of medical images: a survey. IEEE Trans Med Imaging 2003;22:986-1004.

Address:

Eman Shaheen

Department of Oral and Maxillofacial

Surgery

University Hospitals Leuven

Campus Sint-Rafaël

Kapucijnenvoer 33

BE-3000 Leuven

Belgium

Tel: +3216341854

E-mail:eman.shaheen@uzleuven.be 